\title{
HIV Infections: The Global Epidemiology and Goals for Vaccine Research
}

\author{
Giuseppe Ippolito ${ }^{1}$, Giovanni Rezza ${ }^{2}$, Enrico Girardi ${ }^{1}$, Miroslav Malkovsky ${ }^{3}$, \\ and J. Scott Cairns ${ }^{4}$ \\ ${ }^{1}$ Centro di Riferimento AIDS, Servizio di Epidemiologia delle Malattie Infettive \\ IRCCS Ospedale Lazzaro Spallanzani, Rome, Italy \\ ${ }^{2}$ Centro Operativo AIDS, Istituto Superiore di Sanità, Rome, Italy \\ ${ }^{3}$ Department of Medical Microbiology and Immunology, University of Wisconsin \\ Medical School, Madison, Wisconsin, U.S.A. \\ ${ }^{4}$ Targeted Interventions Branch, Division of AIDS, NIAID, Bethesda, Maryland, \\ U.S.A.
}

\section{Introduction}

By mid-1998, more than 30 million people worldwide were living with the human immunodeficiency virus (HIV), with about 5 million of those infected just that year. In the two decades of the HIV epidemic, it has been estimated that over 47 million people were infected with the virus and that nearly 14 million have already died. The epidemic continues to expand, with an estimated doubling time of 10 years. Indeed, the acquired immunodeficiency syndrome (AIDS) has now surpassed tuberculosis and malaria, becoming the leading infectious cause of death. However, patterns of HIV infection and disease are changing. HIV is lowering life expectancy, while reversing previous gains in child survival in east and central Africa, and widespread heterosexual HIV transmission is emerging in parts of southern Africa and southeast Asia. Conversely, AIDS mortality has fallen in industrialized countries, but the prevalence of HIV infection and cost of treatment are increasing (1). The availability of new, effective combinations of anti-retroviral

Address correspondence and reprint requests to: Dr. Miroslav Malkovsky, Department of Medical Microbiology and Immunology, University of Wisconsin Medical School, 1300 University Avenue, Madison, WI 53706, U.S.A. Phone: 608-263-6316. Fax: 608-263-4031; E-mail: mmalkovs@facstaff.wisc.edu drugs is modifying the course of HIV disease, rendering the interpretation of epidemic trends difficult. Despite the fact that successes have been achieved in treating this disease, access to expensive therapies is only possible in the wealthiest nations. The treatments are difficult to administer and commonly fail, thus making prevention strategies of paramount importance for controlling the spread of this deadly virus.

\section{Epidemic Patterns}

Worldwide, an important difference exists between countries with a "concentrated" pattern of HIV transmission and countries with a "generalized" pattern of transmission $(1,2)$. Transmission is considered to be concentrated where the infection is endemic, but occurs mostly in certain population groups, such as male homosexuals or intravenous drug users, which are defined as "high-risk." Regions with a concentrated pattern include western Europe, North America, Australia, and some parts of Latin America and Asia. In these areas, HIV prevalence may be high or very high (from $>5 \%$ to $>50 \%$ ) in affected minorities, though the overall adult prevalence is generally less than $1 \%$ $(1,3)$. In contrast, in the generalized transmission pattern, HIV infection is spread throughout the adult population and heterosexual 
transmission predominates. This is the case in east and central African countries, where the prevalence in adults is well over $1 \%$ (3). In these countries, there may have been a phase of concentrated transmission, possibly attributed to heterosexual transmission among core groups, such as commercial sex workers, truck drivers and businessmen.

In addition, there is a mixed pattern of HIV transmission in some countries due to the coexistence of both concentrated and generalized patterns. This may be the case in countries such as Brazil or India, where the infection began its spread among male homosexuals or female sex workers, after which, generalized transmission radiated into the broader spheres of the population. The comparison between the current "new" global epidemiological pattern and the previously described "old" pattern in which many eastern European and Asian countries were only marginally involved in the epidemic, is presented in Figure 1.

\section{Geographical Variations and Recent Trends}

Geographical variations in the spread of HIV infection are extensive. Six out of every ten HIV-infected men, eight of every ten infected women, and nine in ten infected children live in sub-Saharan Africa, where nearly half of all new infections worldwide are located. HIV incidence in southern Africa rose considerably in the midto late-1990s, reaching record levels in Francistown, Botswana in 1997 and in Beit Bridge, Zimbabwe in 1996 (1). HIV prevalence is also rising in countries such as India, China and Russia, where it was previously low for several years. In particular, in South and Southeast Asia, a region called the "golden triangle" (from eastern Myanmar to Yunnan and Manipur, excepting the Me Kong delta), the pattern of transmission is moving from concentrated (injecting drug users and female sex workers) to generalized transmission. Sex between men is still an important route of infection in Latin America, though heterosexual transmission is common in the Caribbean and generalized transmission is now emerging in some areas of Brazil.

Some evidence indicates declining trends in urban Uganda, where the prevalence of HIV infection in women under 25 years of age attending prenatal centers has been falling since $1994(2,4,5)$. To a lesser extent, some optimistic figures have been obtained in other African countries, such as Zambia $(2,6)$. However, these apparent successes in prevention activity seem to represent the exception rather than the rule in most of the developing world.

The epidemic remains concentrated among minority populations in western countries and in Australia, although in certain urban populations, especially among African Americans in the United States, it has become a generalized phenomenon. In southern Europe, where injecting drug users are heavily affected, heterosexual transmission may play a role in the further spread of HIV. The incidence of AIDS and HIV-related mortality recently fell in most North American and western European countries. However, this does not necessarily reflect an overall decline in the incidence and prevalence of HIV infection. In recent years, progressively more intense anti-retroviral therapies have been introduced into clinical practice, resulting in a decrease of AIDS diagnoses in most developed countries (see below).

\section{HIV Molecular Epidemiology}

The discovery of highly divergent strains of HIV underscores the need for effective surveillance, both for tracking HIV variants and directing research and prevention activities (7). Two major types of HIV have been characterized in human populations: HIV type 1 (HIV-1) - the predominant HIV type throughout the world; and HIV type 2 (HIV-2)-primarily found in persons from West Africa. Although HIV-2 appears to be somewhat less virulent and pathogenic than HIV-1, the clinical disorders induced by HIV-1 and 2 are similar. The genetic variation of HIV is extremely high. There exists a "quasi species" of related, but distinct HIV variants, which vary increasingly over time, not only between hosts, but also even within individual hosts. Rates of mutation and selection pressures vary for different parts of the retroviral genome, but the greatest variation is found in the envelope (env) gene. At least nine subtypes (also called "clades" or "genotypes") of HIV-1 isolates have been classified based on env sequences. These subtypes, designated A through I, constitute the major group of HIV-1, "Group M." Additional strains of HIV-1 that do not belong to Group $M$ have been reported and are referred to as "Group O." They primarily come from Cameroon, where they account for less than 

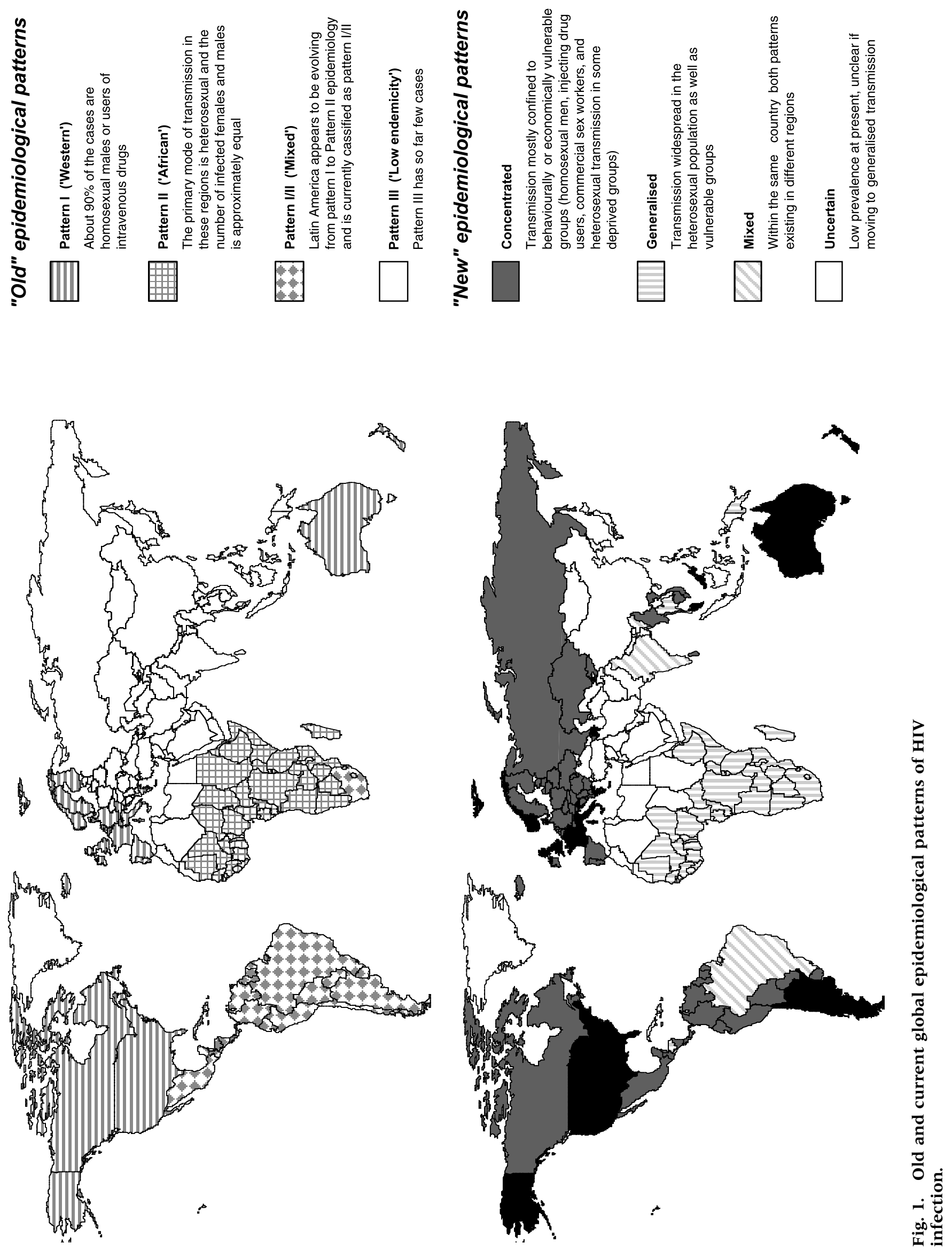
$10 \%$ of HIV infections. Nevertheless, the remaining $90 \%$ of HIV-positive persons (similar to the rest of sub-Saharan Africa) are infected with subtypes $A, C$ and $D$. The highly divergent $\mathrm{O}$ strains might go undetected by commonly used HIV screening tests, raising concerns about test sensitivity for blood transfusion safety as well as for individual diagnosis. Indeed, currently used diagnostic tests are based primarily on subtype B strains, which are predominant in North America and Europe and their sensitivity in detecting divergent strains may not be high.

It is not known whether the genotypic diversity of HIV-1 strains affects transmissibility, infectivity, pathogenicity, or responses to therapy and vaccines. In northern Thailand, subtype $\mathrm{E}$ has been observed to be predominant, where sexual transmission is the main route of HIV transmission. In southern Thailand, type B predominates among injecting drug users. These observations led to the hypothesis that some variants may be more easily transmitted through sexual intercourse than others $(7,8)$. However, this hypothesis has yet to be confirmed. With regard to the role of viral diversity in HIV pathogenesis, different studies show conflicting results and evidence still does not confirm that, after controlling for host factors, different viral strains may determine different progression rates (8). Furthermore, the occurrence and the effect of genetic recombination in persons infected with two different subtypes needs to be evaluated.

In summary, future research should focus on the pathogenicity (length of incubation period) and transmissibility of the different subtypes. It is crucial that we understand more about the host genetic background as a determinant of pathogenicity and transmissibility, cross-immunity between subtypes, and how infections with more than one subtype occur.

\section{Anti-retroviral Treatment and Its Impact}

\section{AIDS Therapies}

Since the introduction of $3^{\prime}$-azido-3'-deoxythymidine (AZT, zidovudine) and its combinations with other nucleoside reverse transcriptase inhibitors (NRTI), such as AZT+3TC, $\mathrm{AZT}+\mathrm{ddI}, \mathrm{AZT}+\mathrm{ddC}$, etc., AIDS chemotherapy has had a profound effect on virus load in HIV- infected persons (9). Especially the triple drug combinations (e.g., NRTI+NRTI+ protease inhibitor (PI), NRTI+PI+PI or NRTI+NRTI+ non-NRTI) are very effective in many patients, even after several years of treatment (9). This treatment is often referred to as "highly active anti-retroviral therapy" (HAART).

\section{Impact of Anti-retroviral Treatment on HIV-related} Morbidity and Mortality

Beginning in 1996, a decrease in the number of new AIDS cases and HIV-related deaths has been observed in several industrialized countries (10-14). The changes are attributed to the increasing use of new anti-retroviral drugs and, in particular, to the increased use of double and triple combination therapies $(10,14)$. Indeed, clinical trials show a higher survival rate for HIV-infected individuals treated with two reverse transcriptase inhibitors, compared with those undergoing monotherapy $(14,15)$, and the highest survival for those treated with triple combination, including protease inhibitors $(14,16,17)$. These data have been confirmed at the population level by observational study results showing decreased risk of AIDSdefining opportunistic infections (14-18) and death (14) over time. It is important to note that these improvements occurred not in a selected population of volunteers who were following a strict protocol, but in a mix of patients who were receiving individualized treatment (19).

The effect of HAART on the epidemic's dynamics in industrialized countries is difficult to predict, because of the number of opposing forces that these treatments may represent relative to virus on transmission and disease progression. With regard to overall HIV prevalence, the prolonged survival of HIV-infected individuals in all stages of disease will tend to increase prevalence; whereas, the diminished viral load of HAART-treated patients may reduce their risk of transmitting the virus, with a corresponding negative effect on the rate of new infections. HAART may also have a dual effect on the prevalence of AIDS. The well-known effect of HAART on increasing the duration of the asymptomatic stage of the disease substantially diminished the rate at which infected individuals are defined as having AIDS. This effect may be counterbalanced by the ability of HAART to prolong survival of persons with AIDS.

Before the introduction of HAART, several studies conducted on cohorts of individuals 
with known HIV seroconversion dates showed that the median time from primary infection to AIDS diagnosis was about 10 years and that the age of infected individuals substantially influenced disease progression. The use of HAART, as well as additional therapies for disorders associated with HIV infections, have modified the clinical course of HIV disease. Longitudinal studies are needed to assess critically the effect of HAART on both the incubation period and the survival time in HIV-infected persons.

\section{Sexual Transmission of HIV:}

\section{Epidemiological Implications}

Sexual transmission continues to account for the vast majority of HIV infections occurring worldwide. Prevention efforts remain focused on modifying sexual behaviors that place people at risk of acquiring HIV infection. Means to reduce the infectivity of HIV-positive persons are being actively investigated. Several lines of evidence support the concept that, regardless of the route of infection, persons with high viral replication are more likely to transmit HIV. As for sexual transmission, studies show that transmission rate is correlated with a lower $\mathrm{CD}^{+}$cell count or a more advanced disease stage $(20,21)$. More recent longitudinal studies further reveal that partners of persons with high plasma viremia are more likely to be infected $(22,23)$. However, to better understand factors associated with sexual transmission, the effects of viral concentration in genital secretions must be evaluated. Numerous studies evaluated the presence of HIV in semen or vaginal secretions by using culture techniques or amplification of viral DNA or RNA. These studies provided evidence that HIV can be frequently recovered from the genital tract. Nonetheless, factors determining viral concentration in genital secretions are not fully understood. In most studies, viral load in genital secretions was higher in persons with higher plasma viremia (24). However, this association was weak. Moreover, some studies observed difference in viral sequences from peripheral blood and genital secretions $(25,26)$. Taken together, these observations suggest that viral concentration in the genital tract does not simply reflect the level of viral replication in blood and that local factors may influence the level of viral replication in the genital tract.

In vitro studies suggest that inflammatory disease of the genital tract may enhance local replication of HIV through a cytokine-mediated mechanism. Further clinical studies showed that the viral burden in genital secretions may be higher in HIV-infected patients with other sexually transmitted diseases (STDs). A study conducted in Malawi showed that HIV-infected men with urethritis had seminal levels of HIV 8 times that of HIV-infected men without urethritis (27). After the urethritis patients received antimicrobial treatment, a sharp decrease in seminal HIV-RNA was observed. In another study conducted among HIV-infected women in Kenya, gonococcal cervicitis and vaginal candidiasis were associated with an increased probability of vaginal HIV shedding (28).

Strategies aimed at reducing the infectivity of HIV-positive persons should include the suppression of viral replication and the identification and reduction of factors that enhance viral replication in the genital tract. Based on the use of three or more drugs, combination anti-retroviral therapies significantly reduce plasma viral load in a high proportion of treated patients. Several studies suggest that a decrease in HIV concentration in genital secretions parallel that recorded in peripheral blood (24). However, replication-competent virus was recovered from seminal cells of patients showing complete suppression of HIV replication in their peripheral blood (29). Therefore, although anti-retroviral therapy probably decreases the infectivity of HIV-positive persons, treated patients must still be considered capable of sexually transmitting HIV. Nevertheless, use of anti-retroviral therapy and the adoption of safer sexual practices may ultimately slow the spread of sexually acquired HIV. Aggressive STD diagnosis and treatment programs may impact further spread of the epidemic by reducing infectivity and probably susceptibility to HIV, both in developing and industrialized countries.

\section{Pediatric AIDS}

Epidemic trends of vertically acquired HIV infection are divergent between industrialized and developing countries. A nearly complete elimination of pediatric AIDS now appears to be an attainable goal in the United States and Europe; whereas, in developing countries, an estimated 1500 HIV-positive children are born each day and many other children are infected soon after birth $(1,2)$. Major reasons for this dif- 
ference include the unavailability of safe alternatives to breast-feeding and lack of access to antiviral drugs that reduce rates of mother-tochild transmission in poorer countries. Giving zidovudine to women during the second and third trimesters of pregnancy, during labor, and to the newborn can reduce vertical transmission of HIV by almost two thirds (30). Widespread, although still incomplete, implementation of this intervention in the United States and in most of Europe has resulted in vertical transmission rates that do not exceed $6 \%(31,32)$ and, among the most recent birth cohorts in industrialized countries, a substantial fall in the incidence of perinatally acquired AIDS.

Available information on HAART (on its safety and how it influences transmission rates) given during pregnancy is limited. Nonetheless, preliminary data suggest that this therapy further reduces transmission rates to an estimated $2 \%$ or less (33). Observational studies (34) and a randomized clinical trial (35) showed that elective cesarean section may reduce vertical transmission rates independent of prophylactic administration of antiviral drugs. Although cesarean section does not appear to be an alternative to anti-retroviral prophylaxis, this intervention may be suitable for HIVinfected women who did not receive antiretroviral drugs during pregnancy (33). Transmission of HIV from mother to child mainly occurs perinatally or postpartum. However, a small percentage of vertical infection may occur during early pregnancy. Access to HIV testing, both before conception and during prenatal care, must be further improved if vertical transmission of HIV infection is to be effectively reduced. The safety and preventive efficacy of HAART administered before pregnancy needs clarification.

The current available drug prophylaxis regimen is too costly and complex for developing countries, where routine cesarean sections and bottle-feeding are also unattainable. These considerations prompted a series of clinical trials evaluating the prophylactic efficacy of zidovudine administered to the mothers during the last weeks of pregnancy (36-38). These simplified regimens appeared to have an efficacy ranging from $50 \%$ for non-breastfeeding mothers to $37 \%$ among breastfeeding mothers. A short zidovudine regimen is much less expensive than the "complete regimen." Even so, its cost is still quite high for the poorer countries and its implementation would require a substantial initial investment to ensure broadened access to HIV testing and prenatal care. Cost-effect analyses showed that short course zidovudine prophylaxis may result in societal savings in most developing countries $(39,40)$.

The results of a more recent trial suggested that improvements in the cost and efficacy of short-term antiviral regimens could be made (41). In this study, $626 \mathrm{HIV}$-infected pregnant women were randomly assigned to one of two groups. One group was given nevirapine orally once at onset of labor and once to their babies within $72 \mathrm{~h}$ of birth. A control group received a standard regimen of zidovudine given orally to the mother at onset of labor and every $3 \mathrm{~h}$ until delivery, and to the infant for $7 \mathrm{~d}$ after birth. Compared with the control group, nevirapine lowered the risk of HIV-1 transmission during the first 14-16 weeks of life by nearly $50 \%$ in this breastfeeding population. Since nevirapine is very inexpensive, its cost should not preclude implementation of this strategy by even the poorest countries and the available data will hopefully foreshadow a dramatic impact on the global epidemic of pediatric AIDS. Implementation of this intervention will require a strong international commitment.

\section{Economic Issues and Anti-retroviral Therapy in Developing Countries: The Unbridgeable Gap}

There is a gap between what is deliverable in the western world and what is affordable where the AIDS burden is heaviest (40). It is sad to say, but HAART will remain unaffordable for most developing countries in the absence of subsidies. Some developing countries, especially in Latin America, may be able to afford some anti-retroviral drugs, but this may not be possible in countries such as Brazil, where HIV is more prevalent (42). Most developing countries have limited resources and cannot provide even the most basic health services. If nothing is done, the gap in access to HAART and other therapies between wealthy countries with controllable disease situations and poor countries with increasing rates of HIV infection will remain unbridgeable.

\section{AIDS Exceptionalism: Privacy Versus Public Health}

Since the beginning of the epidemic, a strategy termed "HIV/AIDS exceptionalism" was 
adopted. This strategy drastically differed from traditional infectious disease control approaches. The policy (based on clinical confidentiality and anonymous surveillance systems, informed consent, and restricted use of HIV antibody tests) did not take into consideration the traditional repressive measures, which consist of routine testing, nominative reporting, contact tracing and notification of those exposed to infected people. Exceptionalism was a safe practice, because it initially had a limited effect on clinical care, with treatments showing only a modest influence on prognosis. The availability of effective treatment should now encourage more widespread HIV testing. Confidential reports of HIV-positive results may also favor a better understanding of the epidemic dynamics in order to better target intervention.

\section{Future Research Priorities in HIV Epidemiology}

Epidemiology of resistance to anti-retroviral drugs is a completely new field. Although the use of combinations of potent anti-retroviral drugs has modified the clinical course of HIV disease (prolonging the global survival of HIVinfected people), less optimistic news comes from reports of the transmission of drug-resistant strains of HIV. Various studies have shown that a relatively high proportion of the viral isolates may have a sharply reduced susceptibility to anti-retroviral drugs and that some patients are infected with multidrug-resistant viruses. As a result, routine resistance testing might be critical for selecting optimal initial treatment regimens or for guiding successive shifts toward other drugs. As HIV-preventive vaccines become available, epidemiologists will be challenged with designing trials in geographic areas with high incidences of HIV infection. This will require the capacity to deal not only with methodological issues, but also with a host of ethical and social concerns that are usually addressed in studies aiming to assess prevention strategies.

The development of HIV surveillance systems is a new challenge. Such systems must be implemented, especially in countries where AIDS incidence has declined as a consequence of the widespread use of effective combination treatment. Problems related to confidentiality and discrimination must be addressed. A backcalculation model that incorporates informa- tion on new HIV diagnosis and time between infection and a certain CD4-level must take the place of surveillance that accounts only for AIDS and its incubation time. An alternative might be a traditional model that assumes variations in the extent of the effect of anti-retroviral treatments on AIDS incubation time.

Systematic molecular testing may provide useful information on circulating viral strains and on changes in their patterns. The predominance of certain strains in geographic areas or in population groups in which a specific transmission pattern exists may contribute to generating hypotheses on strain-related properties. However, ecological associations need to be tested in further analytic studies. Since survival and quality of life have improved, HIVinfected people are likely to live longer, leading normal sex lives. The effect of this phenomenon on sexual transmission of HIV must be evaluated. It remains unknown whether or not increased survival and its effects on transmission are counterbalanced by decreased viral load.

Observational studies are now of great importance to evaluating the population effect of treatment (effectiveness or efficacy in practice) and the long-term effects of anti-retroviral treatment. In fact, though clinical trials remain the best design for evaluating the efficacy of specific drugs or drug regimens, the short study duration and the ideal conditions under which trials are carried out do not permit a thorough evaluation of what will happen in the real world. Observational cohorts will be fundamental for assessing treatment strategies and drug options, and may aid in responding to questions such as: When should anti-retroviral treatment begin? Which is the best regimen to use? At what point should one regimen be substituted with another? All of these questions are crucial in that their answers will determine the future of many HIV-infected individuals, as well as the future of the epidemic. Observational studies may also provide information on long-term toxicity of anti-retroviral drugs and on interruption of treatments.

Population- and hospital-based longitudinal studies are essential if AIDS incidence and HIV-related mortality are to be estimated and changes in the patterns of specific, opportunistic diseases evaluated. This will be of particular use when assessing whether or not HAART affects the occurrence of opportunistic infections and whether it affects the occurrence of 
some more than others. Observational studies may also help us to understand the extent of natural resistance to anti-retroviral drugs, its significance, the incidence of drug-induced resistance, and the extent to which genotypic and/or phenotypic resistance may predict virological, immunological, and clinical outcome.

\section{Goals for HIV Vaccine Research}

Protective vaccination could be the most powerful weapon against the continuous global spread of HIV. Clearly, the most desirable goal for HIV vaccine research would be the development of a vaccine that induces long-term protection against the initial establishment of infection in all vaccinees. Although there are no candidate vaccines close to clinical testing that have been shown to induce this level of protection, some recent advances in AIDS vaccine research offer hope that some objectives of an efficacious AIDS vaccine may ultimately be met. The challenges are formidable and can be attributed, in part, to the nature of the virus and its interaction with the host. HIV tropism for and destruction of cellular subsets that are critical to an effective immune response, as well as the high mutability of the virus, offer unique obstacles that will need to be overcome if a successful AIDS vaccine is to be developed. For vaccine candidates in early stages of development, there are additional issues that need to be considered: which immune responses to target; which delivery system to use; which experimental models to use to test efficacy; which antigens to include in the vaccine preparation. The following section focuses on several of these issues as they relate to preclinical development and testing of HIV vaccine candidates.

\section{Immune Responses to Target}

One of the first issues that needs to be considered in AIDS vaccine development is which immune response to target. Two general classes of specific immunity can potentially be targeted with current vaccine strategies: humoral (antibody) and cellular (T-cell mediated). Antibody $(\mathrm{Ab})$ responses can be further categorized according to isotype and T-cell responses can be defined in terms of surface marker expression and function (CD4 ${ }^{+} /$helpers; $\mathrm{CD}^{+} /$cyto- $^{-}$ toxic $\mathrm{T}$ cells called CTLs). Unfortunately, no firm correlates of protection have been established that allow predictions to be made about the anticipated effectiveness of a vaccine. Studies in primates led to conflicting results about the relative importance of neutralizing $A b$ and CTL responses. In part, this may be a function of the vaccine candidates under study. Different vaccines can induce different types or levels of immune response, which may be protective under certain conditions of challenge. Issues such as the route, dose, or strain of challenge virus, or other features of the host animal, may also determine whether a particular type of immune response will be protective for a given animal. Because of these uncertainties, current vaccine design concepts include as a goal the induction of high levels of broadly reactive neutralizing antibodies and CTL responses, both of which depend on T-cell help for optimal responses.

NEUTRALIZING ANTIBODy. The ability of neutralizing $\mathrm{Ab}$ responses to protect against infection has been investigated in a variety of formats. Many studies have failed to document a role for vaccine-induced neutralizing antibody responses in protection against SIV or SHIV infection in non human primates (43). In humans, control of initial viremia during acute infection does not appear to be mediated by neutralizing Abs; these appear only after the initial viral peak is controlled. Other animal studies, however, point to a potential role for neutralizing Abs in protection from challenge. In several experimental models of HIV infection, passively transferred neutralizing antibodies exerted potent antiviral effects (44-46). Combined, these studies indicated that in certain circumstances, high levels of neutralizing antibody could be protective against viral challenge.

Unfortunately, although it appears that neutralizing antibody could be useful for protection from HIV infection, most vaccine strategies described to date have elicited little or no neutralizing antibody to primary viruses. In particular, recombinant monomeric HIV envelope immunogens elicited $\mathrm{Ab}$ responses that were usually type-specific; did not neutralize primary HIV-1 isolates (47-49); and were not fully protective against infection with viruses that were related, but not identical, to the vaccine strain $(50,51)$. Strategies that can elicit higher levels of $\mathrm{Ab}$ that neutralize a wider variety of primary isolates are, therefore, being 
sought. In one study (52), macaques infected with SIV lacking combinations of three different envelope glycosylation sites (of the approximately 24 sites generally expressed on HIV gp 120) generated markedly increased antibody titers and neutralizing activity against SIV expressing the parental, fully glycosylated form of envelope. Unfortunately, the neutralizing activity was not effective against two other strains of SIV, suggesting that envelope deglycosylation by itself may not induce responses of increased breadth against divergent strains of HIV. When paired with other approaches, however, this strategy may prove useful at augmenting envelope immunogenicity.

Other encouraging results were obtained recently using immunogens that appeared to freeze the viral envelope in a conformation that may exist only after interaction of the virus with cell surface CD4 (53), or CD4 and the chemokine receptor CCR5 (54). In the latter study, a cell-based vaccine elicited $\mathrm{Ab}$ responses in mice that were capable of neutralizing 23/24 primary isolates including $\mathrm{M}$-tropic and T-tropic representatives from five prevalent and geographically distinct HIV clades. Absorption experiments indicated that HIV-1 envelope-specific Abs in the sera were required for the neutralizing effect, providing hope that immunization with envelopes from one or a small number of HIV-1 isolates, when presented in an appropriate conformation, may induce protective responses against a diverse array of primary HIV-1 strains.

CTL. Accumulating evidence has also implicated $\mathrm{CD8}^{+} \mathrm{T}$ cells in the control of initial HIV-1 infection. HIV-specific CD $8^{+}$CTLs were found in the first weeks following infection, before neutralizing antibody appeared, but during the phase when the initial peak of viremia was brought under control $(55,56)$. These results applied to SIV-infected macaques $(57,58)$, where cell-depletion studies more definitively established a role for $\mathrm{CD8}^{+}$cells in controlling the virus (59). In the latter experiments, when chronically SIV-infected macaques were treated with a monoclonal antibody (mAb) to deplete $\mathrm{CD}^{+}$cells, the animals experienced an abrupt rise in plasma viral load that returned to baseline only upon re-emergence of SIV-specific $\mathrm{CD}^{+}$cells. An effect of $\mathrm{CD}^{+}$cell depletion on the viral load of acutely infected animals was also noted. In this instance, animals whose $\mathrm{CD}^{+}$cells were effectively removed by mAb treatment failed to control the initial peak in plasma viremia and experienced an accelerated disease course. The mechanism by which $\mathrm{CD} 8^{+}$ cells control viral replication was unclear in many of these studies: either direct killing of virus-infected cells or control of replication by the release of soluble inhibitory factors, or both, may play a role in viral suppression.

Similar to antibody-mediated viral inhibition, the ability to induce $\mathrm{CD}^{+}$cells that recognize and control different viral isolates is an important consideration in vaccine design. Cases of viral evasion from $\mathrm{CD}^{+}$cell recognition are well documented (60-63) and point to the need to include target antigens with multiple epitopes capable of stimulating a diverse array of $\mathrm{CD}^{+} \mathrm{T}$ cells. To some extent, viral evasion may be less an issue with this arm of the immune response than with Ab. Unlike antibody responses, which need to be focused on exposed regions of the intact virus that are often hypervariable or hidden by glycosylation, CTL responses can be focused on viral epitopes that are not necessarily exposed on the intact virion but are expressed in the infected cell. This allows the possibility of targeting conserved regions of proteins with essential viral functions whose activity would be disrupted by extensive sequence variability (64). Indeed, instances of CTL cross-reactivity against multiple different HIV isolates were documented in volunteers immunized with recombinant canarypox expressing multiple HIV clade Bencoded proteins (65) and in individuals infected with clade B or non-clade B viruses (66). By targeting conserved regions of HIV, the number of strains of HIV that need to be included in a $\mathrm{CD}^{+}$cell-directed vaccine may, thus, be reduced. This will be counterbalanced by the need to include epitopes that are capable of interacting with the array of major histocompatibility (MHC) class I alleles that are present in the vaccinated population. These two counterbalancing forces will dictate the minimal number of epitopes that need to be included in a vaccine to effectively stimulate $\mathrm{CD}^{+}$responses in a genetically heterogeneous population of vaccinees.

HELPER T CELLs. $\mathrm{CD}^{+} \mathrm{T}$ helper (Th) cells have long been known to be important for the generation of $\mathrm{B}$ cell and $\mathrm{CD}^{+}{ }^{+} \mathrm{T}$-cell responses to immunogens and appeared to correlate with slower disease progression in HIV-infected individuals (67). They may also play a direct role 
in controlling HIV replication by secreting IFN- $\gamma, \beta$-chemokines (68) or other viral inhibitory factors, and may themselves kill virusinfected target cells (69). As such, $\mathrm{CD}^{+}{ }^{+}$Th cells play a pivotal role in the immune response and are an obvious consideration in the design of any HIV vaccine candidate. $\mathrm{CD} 4^{+} \mathrm{Th}$ responses are heterogeneous, however, and can stimulate different arms of the immune system depending on the types of cytokines that they produce. Thus, Thl-type responses stimulate CTL responses and humoral responses of the Immunoglobulin G2 (IgG2) subclass; whereas, high levels of IgGl subclass antibodies characterize Th2-type responses. Skewing of Th responses toward Th1-or Th2-type is known to occur in the presence of certain cytokines (70) and, possibly, as a result of interaction with different populations of antigen presenting cells $(71)$ or infectious agents $(72,73)$. This information may prove valuable in the design of vaccines to target particular arms of the immune system.

Although both Th1 and Th2 responses may ultimately be important for the generation of an effective AIDS vaccine, attention focused recently on approaches to selectively stimulate Thl responses. The impetus for this focus was in part, a response to the observation that HIVinfected individuals appeared to switch from a Th1 to a Th2 phenotype as disease progressed (74). Other observations, such as the finding that $\mathrm{CD}^{+}$cell depletion in SIV-infected macaques led to a rapid rise in viral load (59), contributed to the current impression that Th1type responses may be critical components of an immune response elicited by an effective vaccine. Nevertheless, other immune responses, such as the generation of secretory $\operatorname{IgA}(\mathrm{s}-\mathrm{Ig} \mathrm{A})$ in mucosal secretions may play an important role in initial protection from HIV infection and may depend primarily on Th2 responses. If these or other Th2-dependent responses ultimately are found to mediate or improve vaccine-induced protection, optimal vaccines may require the induction of both types of Th responses. Strategies that minimize the mutually antagonistic relationship of these two responses will, therefore, need to be developed.

\section{Mucosal Surfaces}

The mucosal surface is one of the major barriers by which HIV gains access to an uninfected host and is, therefore, an important first site at which to control infection. The cellular composition of this surface has many unusual features that offer unique challenges and opportunities to vaccine strategies targeted to this site. Among the critical issues to address are:

1. Can vaccines targeted at one mucosal surface (e.g., nasal or oral) induce longlasting protective immunity at distant mucosal sites (rectal, vaginal)?

2. What types of mucosal immunity need to be induced to protect against HIV challenge?

3. Does induction of mucosal immunity that is not completely sterilizing have an effect on the level of systemic viral load (and thus disease progression)?

4. Can vaccine-induced mucosal immunity reduce the incidence of HIV transmission by lowering viral load in genital secretions?

The s-IgA molecule is the major humoraldefending factor at mucosal surfaces $(75,76)$. In the human, the amount of s-IgA exported to mucosal surfaces can exceed $3 \mathrm{~g} / \mathrm{d}$, far exceeding the amount of IgG produced in the circulation. Induction of IgA responses is generally considered to be Th-dependent and cytokines characteristic of a Th2 response were associated with increased IgA responses in SIV-infected macaques $(77,78)$, as well as rodents $(79,80)$. This will no doubt be a consideration in primeboost vaccine strategies in which Thl-dependent CTL responses are desired at the same site and time as Th2-dependent IgA responses. In view of the considerable amount of preclinical data suggesting that Thl and Th2 responses are mutually antagonistic, strategies to circumvent this issue by, for instance, the use of different vectors and immunogens given at distinct times or the use of T-independent adjuvants, are of great importance. The observation that high levels of both Th1 and Th2 responses can be generated in certain experimental models of viral infection (81) suggests that this may not an insurmountable problem.

Other cell types commonly found at this site include antigen-specific $\mathrm{T}$ and $\mathrm{B}$ cells. With respect to antigen-specific $\mathrm{CD}^{+}$and $\mathrm{CD}^{+}$ cells, stimulation of these cells at mucosal surfaces occurs through the same set of antigenspecific and co-stimulatory interactions that are required for their stimulation at peripheral 
sites. Additional considerations, including targeting of vaccine components to specialized transport (e.g., $\mathrm{M}$ cells) or antigen presenting cells at these surfaces, may improve the immunogenicity of vaccines delivered to mucosal surfaces.

In addition to antigen-specific $\mathrm{T}$ and $\mathrm{B}$ cells, mucosal surfaces contain a variety of antigen-nonspecific mechanisms, which are the first line of defense in many bacterial and viral infections and which may be considered as potential targets for vaccine-induced interventions. $\gamma-\delta \mathrm{T}$ cells, for instance, are commonly found in mucosal tissues and can be antigen-stimulated to release antiviral factors (82). In addition, many $\mathrm{V} \gamma 9 / \mathrm{V} \gamma 2 \mathrm{~T}$ cells are highly cytotoxic for HIV-infected targets (83). Since stimulation of these cells with their cognate antigens in vivo results in increases in their activity lasting approximately 2 months (84), they may not be the best candidates for vaccine strategies in which the goal is longlasting HIV immunity. However, these and other HIV-nonspecific cells may be good candidates to consider for post-exposure prophylaxis regimens or when the discontinuation of HAART therapy is anticipated. Here, the goal would be to rapidly mobilize an effector population capable of having an impact on the rate of initial viral replication and level of the viral setpoint.

\section{Memory Responses}

Memory $\mathrm{T}$ and B cells are, by definition, those cells that have been previously exposed to their cognate antigen and, as a consequence, are able to respond more quickly and with a heightened response following re-exposure to the same antigen. All efficacious vaccines to date rely for protection on these cells, which are considered critical to the success of an HIV vaccine. In particular, the ability of a vaccine to induce expanded populations of long-lived HIVspecific memory cells will be critical to vaccine success in human populations in parts of the world where access to immunization schemes that demand frequent vaccine boosts may be limited. It appears that $\mathrm{CD}^{+}$memory cells may derive from a relatively rare subset of cells that are activated during primary antiviral responses $(85,86)$. This issue is less clear with respect to $\mathrm{CD} 4^{+} \mathrm{T}$-memory cells, which may persist as activated cells in germinal centers (87). Monitoring these responses will be scientifi- cally daunting, because the markers available to study T-memory cells are not entirely satisfactory.

Regardless of their developmental history, the critical issue for these cells in terms of vaccine design and testing is their ability to protect the host from HIV for prolonged periods of time after vaccination. Currently, most vaccine candidates are tested for protective efficacy during the period of peak vaccine-induced immune activation, but are often not tested after memory cell development. Since HIV vaccines will be expected to induce a state of long-lived immune-mediated protection, vaccines that are advancing through development must be tested for duration of protective response. As such, the ability of a vaccine construct to maintain a pool of potent memory cells will be critical to its success.

\section{Choice of Immunogens}

VIRAL TARGETS. The choice of immunogens to use in a vaccine is, to some extent, dictated by the type of immune response that is sought. Neutralizing antibody responses are primarily directed against envelope proteins, although there is some evidence that antibody responses against Tat may have some beneficial effect in disease progression $(88,89)$. A significant effort is currently underway to determine the structural requirements of an HIV envelope that will allow for induction of high levels of broadly reactive neutralizing responses. As discussed above, recombinant monomeric envelope protein has only succeeded in generating type-specific responses and is, therefore, not likely to be successful in protecting against a broad array of HIV strains. It is not the intent of this discussion to summarize the variety of approaches that are being considered to circumvent this problem, but recent data on the structure of envelope (90) and its interaction with the cell surface (91) will undoubtedly play a large role in formulating strategies to design better envelope-based immunogens.

Other potential viral targets include viral structural, regulatory and accessory gene products. Although neutralizing antibody responses are unlikely to be generated against these targets due to their internal location within infected cells and/or viral particles, CTL and Th epitopes have been mapped to many of these proteins, which may, therefore, form the basis for T-cell based vaccine candidates. There is 
currently no reason to believe that any of these antigens may be qualitatively better in terms of eliciting protective T-cell responses, although attempts to quantify CTL responses to the different HIV proteins in infected individuals suggest that responses to Gag are more frequently found and at higher levels than responses to Env, Pol, or Nef, at least in some patient cohorts $(92,93$, but see 94,95$)$. In addition, the peculiarities of some of these proteins and their interactions with the infected cell suggest that some of these proteins may be better vaccine candidates than others. Recent findings regarding Nef, for instance, provoke caution in targeting this protein as a sole vaccine candidate. Although anti-Nef CTL responses are commonly found in infected individuals, Nef can down-regulate MHC class I molecules on HIV infected cells, thus, protecting them from CTL recognition and lysis (96). Strategies that include Nef as a vaccine component may, therefore, be optimized by deleting regions of this protein responsible for MHC down-regulation or by including Nef-encoded epitopes, rather than whole protein in the vaccine construct.

Epitope-based vaccines are also being considered for HIV. In these approaches, rather than using sequences that encode entire proteins, minimal HIV sequences capable of stimulating individual Th cells, B cells, or CTLs are being derived and tested. Given the combined complications of viral heterogeneity and HLA polymorphism, vaccines that only include single B- or T-cell epitopes are unlikely to have a substantial effect on protecting populations of genetically heterogeneous individuals from HIV infection. Nevertheless, it is conceivable that vaccines that incorporate multiple epitopes from distinct HIV isolates that are restricted to several different HLA alleles may confer protective advantage to the population. Indeed, the finding of HLA "supertypes," which have been defined as related HLA class I and possibly class II alleles that bind peptides with related motifs (97), offers hope that a limited number of HLA class I and class IIrestricted epitopes may be sufficient to induce T-cell responses in a significant percentage of vaccinees. The "linked epitope" approach was investigated using DNA constructs in mice $(98,99)$ and had several attractive features. Constructs can be made in which multiple epitopes are placed in tandem to address issues of viral heterogeneity and HLA polymorphism. In addition, since these constructs will have only minimal homology with the virus itself, the potential for recombination to generate novel and potentially more pathogenic forms of the virus is minimized.

CELlUlar targets. MHC antigens and other adhesion molecules are associated with viral particles. During initial viral exposure, these proteins, derived from the virus donor, could bear antigenic sites, since the virus donor and recipient are genetically different. Indeed, one of the earliest "successes" in nonhuman primate trials of HIV vaccine concepts, in which macaques were immunized and challenged with virus preparations grown in human cells, was shown to depend on the response of the monkeys to human antigens $(100,101)$, a result could be reproduced when macaques were immunized with purified HLADR class II xenoantigens (102). Although the mechanism responsible for protection has not been clearly elucidated yet and attempts to generate protective immune responses using alloantigens, rather than xenoantigens, have been less successful (103), the approach has the advantage in that the immunogen is not susceptible to HIV mutation. Possible disadvantages include the fact that without immediate sterilizing immunity, the vaccine is unlikely to affect subsequent viral load or disease progression since the antigen, provided by the virus donor, will be rapidly lost when host cells in the newly infected recipient begin to replicate the virus.

HIV is also known to require a variety of host cell proteins, in addition to its own viralencoded determinants, for efficient replication. The cellular receptors CD4, CCR5, and CXCR4 are only three of many host cell factors that facilitate cell infection and viral replication. Many of these factors will make inappropriate targets for vaccine development for reasons of self-tolerance or because immune responses to the protein will be deleterious to the individual. However, the discovery of these proteins and the way they interact with HIV, creates novel opportunities to design vaccines that mimic structures created by the interaction of HIV with the host cell. The construction of a "fusion intermediate" vaccine (54) is but one example of the ways in which knowledge of the interaction between host and virus can be used in the creation of novel vaccine candidates. 


\section{Conclusions}

The epidemiological and immunological data discussed above clearly indicate that there are many considerations that must be brought to bear on the preclinical development of an AIDS vaccine. In addition to the issues already discussed, choice of vectors, adjuvants and other formulation issues, routes and timing of delivery, and model systems to use in preclinical testing are additional considerations. Many of these issues have been addressed elsewhere and will not be discussed further here (104107). In addition, once vaccine candidates advance to clinical testing, there are additional issues that must be confronted. Among them, how best to measure efficacy becomes a primary consideration. Indeed, since most current vaccine candidates are unlikely to provide complete, sterilizing immunity to infection, alternative endpoints must be considered in the conduct of clinical trials. These issues have also been extensively discussed (108) and will complicate the design and analysis of largescale clinical trials.

It has been evident for many years now that the development of an effective HIV vaccine is going to be a difficult task that will require the talents, expertise, and imagination of many teams of scientists and clinicians. At a time when the epidemic continues to grow exponentially in many parts of the world, and with millions of people already infected who have no prospect of treatment with today's anti-retroviral regimens, the need for an HIV vaccine has never been greater. Still unclear is how best to induce protective immune responses against a diverse array of HIV isolates in genetically heterogeneous human populations. With advances in our understanding of HIV epidemiology, the interaction of HIV with the host, the structures of HIV-encoded proteins and how they are perceived by the human immune system, and improvements in vaccine delivery systems and formulations, prospects for developing an HIV vaccine are improving.

\section{Acknowledgments}

We thank Drs. Carl Dieffenbach, Nava Sarver, and Paolo Miotti for helpful comments on the manuscript. This work was supported by Ministero della Sanità, Ricerca corrente IRCCS Spallanzani and the NIH.

\section{References}

1. (1998) Monitoring the AIDS pandemic (MAP). The status and trends of the HIVIAIDS epidemics in the world. Geneva MAP, pp. 1-27.

2. Nicoll A, Gill ON. (1999) The global impact of HIV infection and disease. Commun. Dis. Public Health 2: 85-89.

3. Joint United Nations Programme on AIDS (UNAIDS) and the World Health Organization (WHO). Report on the global HIVIAIDS epidemic June 1998. UNAIDS \& WHO, Geneva, pp. 1-58.

4. Stoneburner R, Low-Beer D, Tembo G, Mertens T, Asiimus-Okiror G. (1996) Human immunodeficiency virus dynamics in East Africa deduced from surveillance data. Am. J. Epidemiol. 144: 682-695.

5. Tarantola D, Schwartlander B. (1997) HIV/AIDS epidemics in sub-Saharan Africa: dynamism, diversity and discrete declines? AIDS 11 (suppl B): S5-S21.

6. Fylkesnes K, Musonda RM, Kasumba K, et al. (1997) The HIV epidemic in Zambia: sociodemographic prevalence patterns and indications of trends among childbearing women: AIDS 11: 339-45.

7. Hu DJ, Dondero TJ, Rayfield MA, et al. (1996) The emerging genetic diversity of HIV. The importance of global surveillance for diagnostics, research, and prevention. JAMA 275: 210-2 16.

8. Hu DJ, Buvé A, Baggs J, van der Groen G, Dondero TJ. (1999) What role does HIV-1 subtype play in trasmission and pathogenesis? An epidemiological perspective. AIDS 13: 873-881.

9. Dolin R, Masur H, Saag MS (eds.) (1999) AIDS Therapy. Churchill Livingstone, Philadelphia.

10. CDSC/PHLS: Changes in the incidence of AIDS and in AIDS deaths: the effects of antiretroviral treatment. CDR Wkly 1997, 7: 381.

11. Mouton Y, Alfandari S, Valette M, et al. (1997) Impact of protease inhibitors on AIDS-defining events and hospitalizations in 10 French AIDS reference centres. AIDS 1: F101-F105.

12. Centers for Disease Control and Prevention. (1997) Update: trends in AIDS incidence, deaths, and prevalence-United States, 1996. MMWR 46: 165-173.

13. Hogg RS, O'Shaughnessy MV, Gataric N, et al. (1997) Decline in deaths from AIDS due to new antiretrovirals (letter). Lancet 349: 1294.

14. Pezzotti P, Napoli PA, Acciai S, et al. (1999) Increasing survival time after AIDS in Italy: the role of new combination antiretroviral therapies. AIDS 13: 249-255.

15. Delta Coordinating Committee. (1996) Delta: a randomised double-blind controlled trial comparing combinations of zidovudine plus didanosine or zalcitabine with zidovudine alone in HIV-infected individuals. Lancet 348: 283-291. 
16. Cameron DW, Heath-Chiozzi M, Kravcik S, Mills R, Potthoff A, Henry D. (1996) Prolongation of life and prevention of AIDS complications in advanced HIV immunodeficiency with ritonavir: update. XI International Conference on AIDS. Vancouver, July 1996 vol 1: 24 [abstract MoB411].

17. Hammer SM, Squires KE, Hughes MD, et al. (1997) A controlled trial of two nucleoside analogues plus indinavir in persons with human immunodeficiency virus infection and CD4 cell counts of 200 per cubic millimeter or less. N. Engl. J. Med. 337: 725-733.

18. Brodt HR, Kamps BS, Gute P, Knupp B, Staszewski S, Helm EB. (1997) Changing incidence of AIDS-defining illnesses in the era of antiretroviral combination therapy. AIDS 11: 1731-1738.

19. Hirschel B, Francioli P. (1998) Progress and problems in the fight against AIDS. N. Engl. J. Med. 338: 906-908.

20. Connor EM, Sperling RS, Gelber $R$, et al. (1994) Reduction of maternal-infant transmission of human immunodeficiency virus type 1 with zidovudine treatment. N. Engl. J. Med. 331: 1173-1180.

21. Seage GR, Mayer KH, Horsburgh CR. (1993) Risk of human immunodeficiency virus infection from unprotected receptive anal intercourse increases with decline in immunologic status of infected partners. Am. J. Epidemiol. 137: 899-908.

22. Lee TH, Sakahara N, Fiebig E, Busch MP, O Brien TR, Herman SA. (1996) Correlation of HIV-1 RNA levels in plasma and heterosexual transmission of HIV-1 from infected transfusion recipients. J. Acquir. Immune Defic. Syndr. Hum. Retrov. 12: 427-428.

23. Ragni MV, Faruki H, Kingsley LA. (1998) Heterosexual HIV-1 transmission and viral load in hemophilic patients. J. Acquir. Immune Defic. Syndr. Hum. Retrov. 17: 42-45.

24. Vernazza PL, Eron JJ, Fiscus SA, Cohen MS. (1999) Sexual transmission of HIV: infectiousness and prevention. AIDS 13: 155-166.

25. Zhu T, Zang N, Carr A, et al. (1996) Generic characterization of human immunodeficiency virus type 1 in blood and genital secretions: evidence for viral compartmentalization and selection during sexual transmission. J. Virol. 70: 3098-4107.

26. Coombs RW, Speck CE, Hughes JP, et al. (1998) Association between culturable human immunodeficiency virus type 1 (HIV-l) in semen and blood: evidence for compartmentalization of HIV-1 between semen and blood. J. Infect. Dis. 177: 320-330.

27. Cohen MS, Hoffman IF, Royce RA, et al. (1997) Reduction of concentration of HIV-1 in semen after treatment of urethritis: implications for prevention of sexual transmission of HIV-1. Lancet 349: 1868-1873.

28. Mostad SB, Overbaugh J, DeVange DM, et al. (1997) Hormonal contraception, vitamin A deficiency, and other risk factors for shedding of HIV-1 infected cells from the cervix and vagina. Lancet 350: 922-927.

29. Zhang $H$, Dornadula $G$, Beumont $M$, et al. (1998) Human immunodeficiency virus type 1 in the semen of men receiving highly active antiretroviral therapy. N. Engl. J. Med. 339: 1803-1809.

30. Mayaux MJ, Teglas JP, Mandelbrot L, Berebi A, Gallais H, Matherton S. (1997) Acceptability and impact of zidovudine prevention on mother-to-child HIV 1 transmission in France. J. Paediatr. 131: 857-862.

31. Fiscus SA, Adimora AA, Schoenbach VJ, et al. (1996) Perinatal HIV infection and the effect of zidovudine therapy on transmission in rural and urban counties. JAMA 275: 1483-1488.

32. Stringer JSA, Rouse DJ, Goldenberg RL. (1999) Prophylactic cesarean delivery for the prevention of perinatal human immunodeficiency virus transmission: the case for restraint. JAMA 281: 1946-1949.

33. The International Perinatal HIV Group. (1999) The mode of delivery and the risk of vertical transmission of human immunodeficiency virus type 1: a meta-analysis of 15 prospective cohort studies. N. Engl. J. Med. 340: 977-987.

34. The European Mode of Delivery Collaboration. (1999) Elective caesarean-section versus vaginal delivery in prevention of vertical HIV-1 transmission: a randomised clinical trial. Lancet 353: 1035-1039.

35. Shaffer N, Chuachoowong R, Mock PA, et al. (1999) Short-course zidovudine for perinatal HIV-1 transmission in Bangkok, Thailand: a randomised controlled trial. Lancet 353: 773-780.

36. Wiktor SZ, Ekpini E, Karon JM, et al. (1999) Short course oral zidovudine for prevention of mother-to-child transmission of HIV-1 in Abidjan, Côte d'Ivoire: a randomised trial. Lancet 353: 781-785.

37. Dabis F, Msellati P, Meda N, et al. (1999) 6-month efficacy, tolerance, and acceptability of a short regimen of oral zidovidine to reduce vertical transmission of HIV in breasted children in Côte d'Ivoire and Burkina Faso: a double-blind placebo-controlled multicentre trial. Lancet 353: 786-792.

38. Mansergh G, Haddix AC, Steketee RW, et al. (1996) Cost-effectiveness of short-course zidovudine to prevent perinatal HIV type 1 infection in a sub-Saharan Africa developing country setting. JAMA 276: 139-145.

39. Söderlund N, Zwi K, Kinghorn A, Gray G. (1999) Prevention of vertical transmission of 
HIV: analysis of cost effectiveness of options available in South Africa. BMJ 318: 1650-1656.

40. (1998) AIDS, the unbridgable gap. Lancet 351: 1825.

41. Guay LA, Musoke P, Fleming T, et al. (1999) Intrapartum and neonatal single-dose nevirapine compared with zidovudine for prevention of mother-to-child transmission of HIV-1 in Kampala, Uganda: HIVNET 012 randomized trial. Lancet 354: 795-802.

42. Forsythe S, Gilks C. (1999) Economic issues and antiretroviral therapy in developing countries. Trans. R. Soc. Trop. Med. Hyg. 93: 1-3.

43. Langlois AJ, Desrosiers RC, Lewis MG, et al. (1998) Neutralizing antibodies in sera from macaques immunized with attenuated simian immunodeficiency virus. J. Virol. 72: 69506955.

44. Gauduin MC, Parren PW, Weir R, Barbas CF, Burton DR, Koup RA. (1997) Passive immunization with a human monoclonal antibody protects hu-PBL-SCID mice against challenge by primary isolates of HIV-1. Nat. Med. 3: 1389-1393.

45. Prince AM, Reesink H, Pascual D, et al. (1991) Prevention of HIV infection by passive immunization with HIV immunoglobulin. AIDS Res. Hum. Retro. 7: 971-973.

46. Emini EA, Schleif WA, Nunberg JH, et al. (1992) Prevention of HIV-1 infection in chimpanzees by gp120 V3 domain- specific monoclonal antibody. Nature 355: 728-730.

47. Mascola JR, Snyder SW, Weislow OS, et al. (1996) Immunization with envelope subunit vaccine products elicits neutralizing antibodies against laboratory-adapted but not primary isolates of human immunodeficiency virus type 1. J. Infect. Dis. 173: 340-348.

48. Belshe RB, Graham BS, Keefer MC, et al. (1994) Neutralizing antibodies to HIV-1 in seronegative volunteers immunized with recombinant gp 120 from the MN strain of HIV-1. JAMA 272: 475-480.

49. Belshe RB, Gorse GJ, Mulligan MJ, et al. (1998) Induction of immune responses to HIV1 by canarypox virus (ALVAC) HIV-l and gp120 SF-2 recombinant vaccines in uninfected volunteers. AIDS 12: 2407-2415.

50. Belshe RB, Bolognesi DP, Clements ML, et al. (1994) HIV infection in vaccinated volunteers. JAMA 272: 431.

51. Kahn JO, Steimer KS, Baenziger J, et al. (1995) Clinical, immunologic, and virologic observations related to human immunodeficiency virus (HIV) type 1 infection in a volunteer in an HIV- 1 vaccine clinical trial. J. Infect. Dis. 171: 1343-1347.

52. Reitter JN, Means RE, Desrosiers RC. (1998) A role for carbohydrates in immune evasion in AIDS. Nat. Med. 4: 679-684.
53. Devico A, Silver A, Thronton AM, Sarngadharan MG, Pal R. (1996) Covalently crosslinked complexes of human immunodeficiency virus type 1 (HIV-1) gp120 and CD4 receptor elicit a neutralizing immune response that includes antibodies selective for primary virus isolates. Virology 218: 258-263.

54. LaCasse RA, Follis KE, Trahey M, Scarborough JD, Littman DR, Nunberg JH. (1999) Fusioncompetent vaccines: broad neutralization of primary isolates of HIV. Science 283: 357-362.

55. Koup R. A., Safrit J. T., Cao Y., et al. (1994) Temporal association of cellular immune responses with the initial control of viremia in primary human immunodeficiency virus type 1 syndrome. J. Virol. 68: 4650-4655.

56. Borrow P, Lewicki H, Hahn BH, Shaw GM, Oldstone MBA. (1994) Virus-specific CD8+ cytotoxic T-lymphocyte activity associated with control of viremia in primary human immunodeficiency virus type 1 infection. J. Virol. 68: 6103-6110.

57. Yasutomi Y, Reimann KA, Lord CI, Miller MD, Letvin NL. (1993) Simian immunodeficiency virus-specific CD8 $+\mathrm{T}$ lymphocyte response in acutely infected rhesus monkeys. J. Virol. 67: 1707-1711.

58. Kuroda MJ, Schmitz JE, Charini WA, et al. (1999) Emergence of CTL coincides with clearance of virus during primary simian immunodeficiency virus infection in rhesus monkeys. J. Immunol. 162: 5127-5133.

59. Schmitz JE, Kuroda MJ, Santra S, et al. (1999) Control of viremia in simian immunodeficiency virus infection by $\mathrm{CD}^{+}$lymphocytes. Science 283: 857-860.

60. Price DA, Goulder PJ, Klenerman $\mathrm{P}$, et al. (1997) Positive selection of HIV-1 cytotoxic T lymphocyte escape variants during primary infection. Proc. Natl. Acad. Sci. U.S.A. 94: 1890-1895.

61. Goulder PJ, Phillips RE, Colbert RA, et al. (1997) Late escape from an immunodominant cytotoxic T-lymphocyte response associated with progression to AIDS. Nat. Med. 3: 212-217.

62. Borrow P, Lewicki H, Wei X, et al. (1997) Antiviral pressure exerted by HIV-1-specific cytotoxic T lymphocytes (CTLs) during primary infection demonstrated by rapid selection of CTL escape virus. Nat. Med. 3: 205-211.

63. Wilson CC, Brown RC, Korber BT, et al. (1999) Frequent detection of escape from cytotoxic Tlymphocyte recognition in perinatal human immunodeficiency virus (HIV) type 1 transmission: the ariel project for the prevention of transmission of HIV from mother to infant. J. Virol. 73: 3975-3985.

64. Wagner R, Leschonsky B, Harrer E, et al. (1999) Molecular and functional analysis of a 
conserved CTL epitope in HIV-1 p24 recognized from a long-term nonprogressor: constraints on immune escape associated with targeting a sequence essential for viral replication. J. Immunol. 162: 3727-3734.

65. Ferrari G, Humphrey W, McElrath MJ, et al. (1997) Clade B-based HIV-1 vaccines elicit cross-clade cytotoxic $\mathrm{T}$ lymphocyte reactivities in uninfected volunteers. Proc. Natl. Acad. Sci. U.S.A. 94: 1396-1401.

66. Cao H, Kanki P, Sankale JL, et al. (1997) Cytotoxic T-lymphocyte cross-reactivity among different human immunodeficiency virus type 1 clades: implications for vaccine development. J. Virol. 71: 8615-8623.

67. Rosenberg ES, Billingsley JM, Caliendo AM, et al. (1997) Vigorous HIV-1-specific CD4+ T cell responses associated with control of viremia. Science 278: 1447-1450.

68. Schrum S, Probst P, Fleischer B, Zipfel PF. (1996) Synthesis of the CC-chemokines MIP$1 \alpha$, MIP- $1 \beta$, and RANTES is associated with a type 1 immune response. J. Immunol. 157: 3598-3604.

69. Miskovsky E. P., Liu A. Y., Pavlat W., et al. (1994) Studies of the mechanism of cytolysis by HIV-1-specific CD4+ human CTL clones induced by candidate AIDS vaccines. J. Immunol. 153: 2787-2799.

70. Paul WE, Seder RA. (1994) Lymphocyte responses and cytokines. Cell 76: 241-251.

71. Rissoan MC., Soumelis V., Kadowaki N., et al. (1999) Reciprocal control of T helper cell and dendritic cell differentiation. Science 283: 1183-1186.

72. Doherty TM, Coffman RL. (1996) Leishmania major: effect of infectious dose on $\mathrm{T}$ cell subset development in BALB/c mice. Exp. Parasitol. 84: 124-135.

73. Sieling PA, Wang XH, Gately MK, et al. (1994) IL-12 regulates $\mathrm{T}$ helper type $\mathrm{l}$ cytokine responses in human infectious disease. J. Immunol. 153: 3639-3647.

74. Cohen J. (1993) T cell shift: key to AIDS therapy? Science 262: 175-176.

75. Mestecky J, McGhee JR. (1987) Immunoglobulin IgA: molecular and cellular interactions involved in IgA biosynthesis and immune response. Adv. Immunol. 40: 153-245.

76. Kraehenbuhl, JP, Neutra MR. (1992) Molecular and cellular basis of immune protection of mucosal surfaces. Physiological Rev. 72: 853-879.

77. Kawabata S, Miller CJ, Lehner T, et al. (1998) Induction of Th2 cytokine expression for p27specific IgA B cell responses after targeted lymph node immunization with simian immunodeficiency virus antigens in rhesus macaques. J. Infect. Dis. 177: 26-33.

78. McGhee JR, Kiyono H, Kubota M, et al. (1999) Mucosal Th1- versus Th2-type responses for antibody- or cell-mediated immunity to simian immunodeficiency virus in rhesus macaques. J. Infect. Dis. 179 (Suppl 3): S480-S484.

79. Petitprez K, Khalife J, Cetre C, et al. (1999) Cytokine mRNA expression in lymphoid organs associated with the expression of IgA response in the rat. Scand. J. Immunol. 49: 14-20.

80. Yamamoto $S$, Kiyono $H$, Yamamoto $M$, et al. (1997) A nontoxic mutant of cholera toxin elicits Th2-type responses for enhanced mucosal immunity. Proc. Natl. Acad. Sci. U.S.A. 94: 5267-5272.

81. Whitmire JK, Asano MS, Murali-Krishna K, Suresh M, Ahmed R. (1998) Long-term CD4 Th1 and Th2 memory following acute lymphocytic choriomeningitis virus infection. J. Virol. 72: 8281-8288.

82. Poccia F, Battistini L, Cipriani B, et al. (1999) Phosphoantigen-reactive $\mathrm{V} \gamma 9 \mathrm{~V} \delta 2 \mathrm{~T}$ lymphocytes suppress in vitro human immunodeficiency virus type 1 replication by cell-released antiviral factors including CC chemokines. J. Infect. Dis. 180: 858-861.

83. Wallace M, Bartz SR, Chang W-L, MacKenzie D, Pauza CD, Malkovsky M. (1996) $\gamma \delta$ T lymphocytes responses to human immunodeficiency virus. Clin. Exp. Immunol. 103: 177-184.

84. Poccia F, Malkovsky M, Pollak A, et al. (1999) In vivo $\gamma \delta \mathrm{T}$-cell priming to mycobacterial antigens by primary $M$. tuberculosis infection and exposure to nonpeptidic ligands. Mol. Med. 5: 471-476.

85. Jacob J, Baltimore D. (1999) Modeling T-cell memory by genetic marking of memory $\mathrm{T}$ cells in vivo. Nature 399: 593-597.

86. Sourdive DJ, Murali-Krishna K, Altman JD, et al. (1998) Conserved $\mathrm{T}$ cell receptor repertoire in primary and memory CD8 $\mathrm{T}$ cell responses to an acute viral infection. J. Exp. Med. 188: 71-82.

87. Castan J, Tenner R, Racz P, Fleischer B, Broker BM. (1997) Accumulation of CTLA-4-expressing $\mathrm{T}$ lymphocytes in the germinal centres of human lymphoid tissues. Immunology 90: 265-271.

88. Re MC, Furlini G, Vignoli M, et al. (1995) Effect of antibody to HIV-1 Tat protein on viral replication in vitro and progression of HIV-1 disease in vivo. J. Acquir. Immune Defic. Syndr. Hum. Retrovirol. 10: 408-416.

89. Zagury JF, Sill A, Blattner W, et al. (1998) Antibodies to the HIV-1 Tat protein correlated with nonprogression to AIDS: a rationale for the use of Tat toxoid as an HIV-l vaccine. J. Hum. Virol. 1: 282-292.

90. Kwong PD, Wyatt R, Robinson J, Sweet RW, Sodroski J, Hendrickson WA. (1998) Structure of an HIV gp120 envelope glycoprotein in complex with the CD4 receptor and a neutralizing human antibody. Nature 393: 648-659. 
91. D'Souza MP, Harden VA. (1996) Chemokines and HIV-1 second receptors. Confluence of two fields generates optimism in AIDS research. Nat. Med. 2: 1293-1300.

92. Lynch JA, deSouza M, Robb MD, et al. (1998) Cross-clade cytotoxic $T$ cell response to human immunodeficiency virus type 1 proteins among HLA disparate North Americans and Thais. J. Infect. Dis. 178: 1040-1046.

93. Bernard NF, Pederson K, Chung F, Ouellet L, Wainberg MA, Tsoukas CM. (1998) HIV-specific cytotoxic T-lymphocyte activity in immunologically normal HIV-infected persons. AIDS 12: 2125-2139.

94. Chenciner N, Michel F, Dadaglio G, et al. (1989) Multiple subsets of HIV-specific cytotoxic $\mathrm{T}$ lymphocytes in humans and in mice. Eur. J. Immunol. 19: 1537-1544.

95. Larsson M., Jin X., Ramratnam B., et al. (1999) A recombinant vaccinia virus based ELISPOT assay detects high frequencies of Pol-specific CD8 $\mathrm{T}$ cells in HIV-1-positive individuals. AIDS 13: 767-777.

96. Collins KL, Chen BK, Kalams SA, Walker BD, Baltimore D. (1998) HIV-1 Nef protein protects infected primary cells against killing by cytotoxic T lymphocytes. Nature 391: 397-401.

97. Sette A, Sidney J. (1998) HLA supertypes and supermotifs: a functional perspective on HLA polymorphism. Curr. Opin. Immunol. 10: 478-482.

98. Thomson SA, Sherritt MA, Medveczky J, et al. (1998) Delivery of multiple CD8 cytotoxic T cell epitopes by DNA vaccination. J. Immunol. 160: 1717-1723.

99. Woodberry T, Gardner J, Mateo L, et al. (1999) Immunogenicity of a human immunodeficiency virus (HIV) polytope vaccine containing multiple HLA A2 HIV CD8(+) cytotoxic T-cell epitopes. J. Virol. 73: 5320-5325.

100. Stott EJ, Chan WL, Mills KH, et al. (1990) Preliminary report: protection of cynomolgus macaques against simian immunodeficiency virus by fixed infected-cell vaccine. Lancet 336: 1538-1541.

101. Cranage MP, Polyanskaya N, McBride B, et al. (1993) Studies on the specificity of the vaccine effect elicited by inactivated simian immunodeficiency virus. AIDS Res. Hum. Retroviruses 9: 13-22.

102. Arthur LO, Bess JWJr, Urban RG, et al. (1995) Macaques immunized with HLA-DR are protected from challenge with simian immunodeficiency virus. J. Virol. 69: 3117-3124.

103. Polyanskaya N, Sharpe S, Cook N, et al. (1997) Anti-major histocompatibility complex antibody responses to simian B cells do not protect macaques against SIVmac infection. AIDS Res. Hum. Retroviruses 13: 923-931.

104. Cairns JS, Sarver N. (1998) New viral vectors for HIV vaccine delivery. AIDS Res. Hum. Retroviruses 14: 1501-1508.

105. Stott EJ, Schild GC. (1996) Strategies for AIDS vaccines. J. Antimicrob. Chemother. 37 (Suppl B): 185-98.

106. Vogel FR. (1998) Adjuvants in perspective. Dev. Biol. Stand. 92: 241-248.

107. Alving CR, Detrick B, Richards RL, Lewis MG, Shafferman A, Eddy GA. (1993) Novel adjuvant strategies for experimental malaria and AIDS vaccines. Ann. N.Y. Acad. Sci. 690: 265-275.

108. Hoff R, Barker LF. (1995) Trial objectives and end points for measuring the efficacy of HIV vaccines. Infect. Agents Dis. 4: 95-101. 